\section{The influence of interpersonal comparisons of outcomes under conditions of free responding*}

\author{
THOMAS L. RADINSKY† and SURESH KANEKAR †† \\ University of Iowa, Iowa City, Iowa 52240
}

A mutual fate control (MFC) situation is one in which two persons control the positive and negative outcomes of each other. Studies of the MFC and related situations indicated that the attainment of mutually positive outcomes is generally impeded when individuals are given explicit, trial-by-trial information about both their own and the other person's outcomes. In these studies, the choosers' responses were externally paced. In the present study, the choosers were permitted to respond at will. The results of the present study were consistent with those of the paced response studies. Showing individuals both their own and the other person's outcomes resulted in significantly fewer positive outcomes than showing individuals only their own outcomes. These results suggest that the opportunity to make outcome comparisons led to the dyad members' doing more poorly, in terms of the choosers' own outcomes, than when individuals were experimentally prohibited from making such comparisons.

Typically, when individuals interact with one another, the satisfaction of each person involved in the interaction is dependent upon both his own and the others' actions. In order to study factors which influence interpersonal interaction under controlled conditions, social psychologists have frequently employed the experimental game paradigm.

Within the experimental game paradigm, as it is generally used, the pacing of the choosers' responses is externally timed. Dyad members are given a cue that indicates when to respond, and feedback is given to the individuals only after both have responded. This situation probably occurs infrequently in the natural environment, and the present experiment represents a further extension of the experimental game research into a free response situation.

The situation investigated in the present study is that designated as one of mutual fate control (MFC) by Thibaut \& Kelley (1959). In this situation, each of two $S$ s is typically restricted to two responses, one of which provides a positive and the

*This study was conducted at Cornell College, Mt. Vernon, Iowa, during the second author's tenure there as Part-time Visiting Lecturer in Psychology. The help of David A. Bernstein, Acting Chairman of the Department of Psychology, Cornell College, in making space and $S s$ available, and the assistance of John Greenwood, in running the experiment, are gratefully acknowledged.

+Now at the American Institutes for Research, Pittsburgh, $\mathrm{Pa}$. Requests for reprints should be sent to the American Institutes for Research, 710 Chatham Center, Pittsburgh, Pa. 15219. India. other a negative consequence to the other person. In the MFC situation, there are three possible joint states: (1) mutual positive outcomes, (2) mutual negative outcomes, and (3) positive outcome for one person and negative outcome for the other.

Of interest in the present study was whether, under conditions of free responding, providing information about both the chooser's own and the other person's outcomes would facilitate or impede attainment of joint positive outcomes relative to providing information only about the chooser's own outcomes.

Kelley and his associates (e.g., Kelley, 1968; Kelley, Thibaut, Radloff, \& Mundy, 1962; Rabinowitz, Kelley, \& Rosenblatt, 1966; Thibaut \& Kelley, 1959) suggest that the principal problem in the MFC situation is one of response coordination, since there exists a response combination which gives both parties their maximum reward simultaneously (Kelley, 1968). Insofar as the main MFC problem is one of response coordination, supplying more information about the situation is expected to facilitate attainment of positive outcomes. This interpretation has been supported in experiments by Kelley et al (1962) and Smith (1968) in which Ss' responses were paced.

However, not all types of information may be beneficial. A factor that may result in a reduction in positive outcomes is outcome comparison. If an individual has information available about both his own and the other person's outcomes, he may attempt to outscore (or avoid being outscored by) the other person. In order to achieve this goal in the
MFC situation, the chooser must provide a negative outcome for the other person while the other person provides a positive outcome for the chooser. Thus, if individuals attempt to outscore one another, they are likely to provide each other with negative outcomes. Evidence, obtained under conditions of paced responding, is available to support this analysis (see, for example, Messick \& Thorngate, 1967; Rapoport, 1969; Radinsky, 1969).

\section{METHOD}

The Ss $(N=32)$ were introductory psychology students at Cornell College who agreed to participate to fulfill course requirements. Two $S s$, seated in different rooms separated by a hallway, participated in each session. In general, the Ss arrived at different times, and consequently did not see one another prior to the experiment. No close friends participated in the same session. The dyad members were not restricted to the same sex.

When a $\mathbf{S}$ arrived, he was seated at a table on which was placed an unlabeled two-position switch. To the right of this switch was a vertical bank of four four-digit pulse counters, all set on zero. The counters were alternatively labeled "Pos. Points" and "Neg. Points" from top to bottom. To the right of the counters was a panel. For half the Ss, the top two counters were designated "Your Points," and the bottom two counters were unlabeled and remained at zero throughout the experiment. For the other half of the Ss, the label "Other's Points" was added to the bottom two counters, and all four counters functioned during the experiment.

All Ss were told that two persons would participate in the experiment at the same time, but no other information was given about the nature of the interdependence or how the outcomes were obtained. They were also told that their switch had two positions and that they could move it from one position to the other whenever they wished. It was stated that the S's task was "to get as many positive points and as few negative points" as he could. These points had no monetary value. Finally, the Ss were instructed not to talk, and were given an opportunity to ask questions, which were answered by paraphrasing the instructions.

Since a direct extension of a paced MFC paradigm to one of free responding would introduce a third response, that of not making either response, a technique similar to one adopted by Rabinowitz et al (1966) was employed in the present study. With this technique, one position of a chooser's switch produced a state (for the other person) which persisted until 


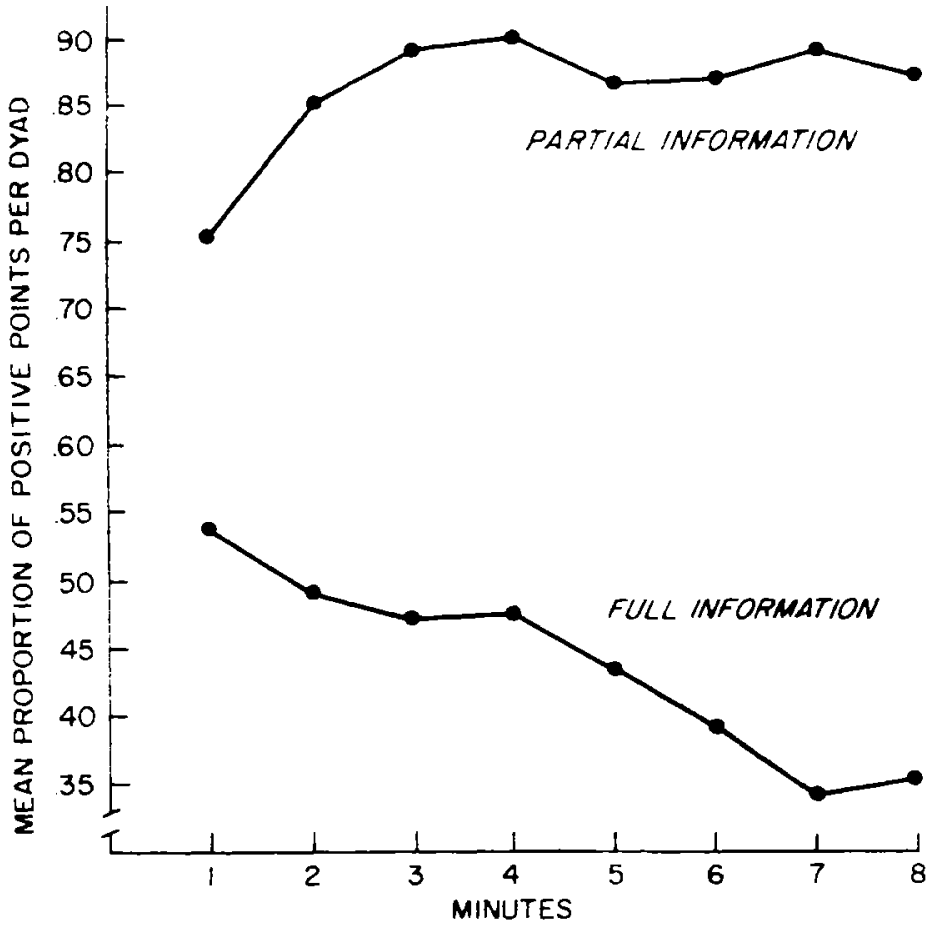

Fig. 1. Proportion of positive points per dyad in each minute as a function of information.

the switch was changed to the other position. Each individual was thereby always receiving either positive or negative outcomes.

Specifically, a pair of interconnected interval timers was used to generate a brief pulse about every $1 / 2$ sec. The Ss' switches directed these pulses to the other person's positive or negative pulse counters. The experimental task lasted $8 \mathrm{~min}$. The $E$ had a set of counters from which a reading was taken at $1-\mathrm{min}$ intervals.

After the timers were shut off, the Ss were interviewed.

\section{RESULTS}

Figure 1 shows the mean proportion of positive points to the total number of points received by each dyad in each 1 -min interval. A 2 by 8 analysis of variance, with a between factor of information and a within factor of minutes, was performed using the proportion of positive points per dyad per minute as a dependent variable. There was a significant effect of information $(F=17.31, \mathrm{df}=1 / 14$, $p<.001)$. Dyads given partial information had a much higher proportion of positive points in all minutes than did dyads given full information. In addition, there was a significant Information by Minutes interaction $(\mathrm{F}=2.58, \mathrm{df}=7 / 98$, $\mathrm{p}<.05$ ). Inspection of Fig. 1 reveals that this interaction stems from an increase over time in the proportion of positive points for the partial information dyads and a decrease for the full information dyads.

\section{DISCUSSION}

The results of the present paced response condition, individuals make and are influenced by own and the other person's outcomes. As was true of the situation used by outcome comparisons led to the dyad members' doing more poorly, in terms of the choosers' own outcomes, than individuals who were experimentally prohibited from making such comparisons. Further analysis of the results of the present experiment indicates that free responding experiment suggest that, as in the comparisons they make between their Messick \& Thorngate (1967), these individuals, viewing only their own outcomes, tend to remain in the mutually positive outcome state once it is attained. As a group, they attained mean levels of positive outcomes as high as or higher than those reported in MFC studies where $S$ responses were paced (e.g., Kelley et al, 1962; Rabinowitz et al, 1966; Smith, 1968).

The common occurrence of free response relationships between people in the natural environment would appear to justify additional research on several factors using the free response paradigm. One factor is that of incentive magnitude. In the present experiment, the magnitude of the incentives was relatively low. Shock and money have often been used for negative and positive outcomes. Whether less concern with differences in outcomes would be exhibited with incentives of greater magnitude is a question in need of investigation. Also in need of further study is the behavior of individuals under conditions of free responding in situations having a structure different from that of the MFC situation. Some work along these lines has been done by Rabinowitz et al (1966), but considerably more work is needed.

\section{REFERENCES}

K EL LEY , H . H. Interpersona ? accommodation. American Psychologist, 1968, 23, 399-410.

KELLEY, H. H., THIBAUT, J. W., RADLCFF, R., \& MUNDY, D, The development of cooperation in the "minimal social situation." Psychological Monographs, 1962, 76(19, Whole No. 538).

MESSICK, D. M., \& THORNGATE, W. B Relative gain maximization in experimental games. Journal of Experimental Social Psychology, 1967, 3. 85-101.

RABINOWITZ, L., KELLEY, H, H., \& ROSENBLATT, R. M. Effects of different types of interdependence and response conditions in the minimal social situation. Journal of Experimental Social Psychology, 1966, 2, 169-197.

RADINSKY, T. L. Equity and inequity as a source of reward and punishment. Psychonomic Science, 1969, 15. 293-294.

RAPOPORT, A. Effects of payoff information in multistage mixed-motive game. Behavioral Science, 1969,14, 204-215.

SMITH, W. P. Reward structure and information in the development of cooperation. Journal of Experimental Social Psychology, 1968, 4, 199-223.

THIBAUT, J. W., \& KELLEY, H. H, The social psychology of groups. New York: Wiley, 1959 . 